\title{
Über die Vollständigkeit verallgemeinerter freier Felder in einer Zeitschicht
}

\author{
Claus Möllenhoff \\ Institut für Theoretische Physik der Universität Göttingen \\ Eingegangen am 16. August 1968
}

\begin{abstract}
A necessary and sufficient condition for the completeness of generalized free fields in a time-slice is established.
\end{abstract}

\section{Einleitung}

Es sei $T$ eine Zeitschicht im vierdimensionalen Minkowskiraum, d. h.

$$
T=\left\{x:\left|x_{0}\right|<\left|t_{0}\right|\right\} .
$$

Dann erfüllt ein Feld $A(f)$ das Zeitschichtpostulat, (ZSP), falls für einen beliebigen beschränkten Operator $C$ gilt:

Aus $[A(f), C]=0$ für supp $f \subset T$

folgt $[A(g), C]=0$ für suppg beliebig.

Das heißt mit anderen Worten: Die ganze Algebra der Feldoperatoren läßt sich durch Operatoren erzeugen, deren Testfunktionen ihren Träger in $T$ haben (Vollständigkeit).

Das verallgemeinerte freie Feld wird festgelegt durch ein positives, lorentzinvariantes $\mathrm{Maß} \mu(p)$ (z. B. [3], [4]). H. J. Borchers zeigte in [1], daß das ZSP gilt, wenn für $\mu(p)$

$$
\int e^{\lambda \sqrt{p^{2}}} d \mu(p)<\infty, \quad \lambda>0
$$

erfüllt ist. HAAG und Schroer zeigten in [2], daß das ZSP nicht gilt, wenn $\mu(p)$ im Unendlichen langsamer oder ebenso abfällt wie

$$
\frac{1}{(1+|p|)^{k}}, \quad k=0,1,2, \ldots, n<\infty .
$$

Im folgenden soll eine notwendige und hinreichende Bedingung für die Gültigkeit des ZSP in Abhängigkeit von $\mu(p)$ gegeben werden.

\section{Ergebnisse}

Es sei $T$ eine Zeitschicht, $C$ sei ein beliebiger beschränkter Operator des Fockraumes

$$
\mathscr{H}=\bigoplus_{n=0}^{\infty} \mathscr{H}_{n} .
$$


$A(f)$ sei ein verallgemeinertes freies Feld, definiert durch das Maß $\mu(p)$. Wir betrachten die Menge $\mathscr{D}(T)$ der Testfunktionen mit kompaktem Träger in $T$. Thre Fouriertransformationen seien mit $\tilde{\mathscr{D}}(T)$ bezeichnet, also

Weiterhin sei

$$
f(x) \in \mathscr{D}(T), \quad \tilde{f}(p) \in \tilde{\mathscr{D}}(T)
$$

Dann gilt das folgende

$$
\mu_{1}(p)=\mu(p)+\mu(-p) .
$$

Theorem 1. Das Zeitschichtpostulat gilt dann und nur dann für das verallgemeinerte freie Feld $A(f)$, wenn $\tilde{\mathscr{D}}(T)$ dicht in $\mathscr{L}_{2}\left(\mu_{1}\right)$ ist.

Aus Theorem 1 ist ersichtlich, daß das ZSP höchstens dann gelten kann, wenn $\mu(p)$ stärker als jedes Polynom abfällt. Aus diesem Grunde sind solche Felder und ihre zeitlichen Ableitungen zu einer scharfen Zeit wohldefiniert. Es ergibt sich folgende Verschärfung:

Theorem 2. Gilt das ZSP für eine Zeitschicht der Dicke $\left|t_{0}\right|>0$, so gilt es auch noch für eine $\delta$-förmige Zeitschicht.

\section{Beweise}

Zum Beweis unserer Theoreme formulieren wir mehrere Hilfssätze:

Lemma 1. Sei $\tilde{\varphi}$ beliebig $\in \mathscr{L}_{2}\left(\mu_{1}\right)$. Aus $[\widetilde{A}(\tilde{f}), \widetilde{A}(\tilde{\varphi})]=0$ für $\tilde{f}(p) \in \tilde{\mathscr{D}}(T)$ folgt $[\widetilde{A}(\tilde{g}), \widetilde{A}(\tilde{\varphi})]=0$ für alle $\tilde{g} \in \mathscr{L}_{2}\left(\mu_{1}\right)$ dann und nur dann, wenn $\tilde{\mathscr{D}}(T)$ dicht in $\mathscr{L}_{2}\left(\mu_{1}\right)$ ist.

Beweis. Es ist (s. [1])

$$
(\Omega,[\widetilde{A}(\tilde{f}), \widetilde{A}(\tilde{\varphi})] \Omega)=[c-\mathrm{Zahl}](\Omega, \Omega) .
$$

Statt der Kommutatoren können wir also auch deren Vakuumerwartungswerte betrachten.

a) Aus $[\widetilde{A}(\tilde{)}), \widetilde{A}(\tilde{\varphi})]=0$ möge $[\widetilde{A}(\tilde{g}), \widetilde{A}(\tilde{\varphi})]=0$ folgen. Für $\widetilde{A}$ gilt nach Definition (s. [4])

Also

$$
\widetilde{A}(\tilde{g}(p)) \Omega=\tilde{g}(p) \in \mathscr{H}_{1} .
$$

$$
\begin{aligned}
(\Omega,[\widetilde{A}(\tilde{f}), \widetilde{A}(\tilde{\varphi})] \Omega) & =(\Omega, \tilde{A}(\tilde{f}) \tilde{\varphi})-(\Omega, \widetilde{A}(\tilde{\varphi}) \tilde{f}) \\
& =\int \tilde{f}(-p) \tilde{\varphi}(p) d \mu(p)-\int \tilde{\varphi}(-p) \tilde{f}(p) d \mu(p) \\
& =\int \tilde{f}(p)\{\tilde{\varphi}(-p) \Theta(-p)-\tilde{\varphi}(-p) \Theta(p)\} d \mu_{\mathbf{1}}(p) \\
& =\int \tilde{f}(p) \tilde{\varphi}^{\prime}(p) d \mu_{1}=0
\end{aligned}
$$

mit $\tilde{\varphi}^{\prime} \in \mathscr{L}_{2}\left(\mu_{1}\right)$ und $\tilde{f} \in \tilde{\mathscr{D}}(T)$.

Ebenso ist

$$
[\widetilde{A}(\tilde{g}), \widetilde{A}(\tilde{\varphi})]=\int \tilde{g}(p) \tilde{\varphi}^{\prime}(p) d \mu_{1}=0
$$


für alle $\tilde{g} \in \mathscr{L}_{2}\left(\mu_{1}\right)$. Diese Integrale lassen sich als Skalarprodukt im $\mathscr{L}_{2}\left(\mu_{1}\right)$ auffassen. Da $\tilde{\varphi}$ und damit $\tilde{\varphi}^{\prime}$ beliebig gewählt waren, ist also $\tilde{\mathscr{D}}(T)$ dicht in $\mathscr{L}_{2}\left(\mu_{1}\right)$.

b) Die Gegenrichtung ist trivial.

Lemma 2. Aus $[A(f), C]=0$ für $f \in \mathscr{D}(T)$ folgt $(\Omega,[A(g), C] \Omega)=0$ für suppg beliebig kompakt dann und nur dann, wenn $\tilde{\mathscr{D}}(T)$ dicht in $\mathscr{L}_{2}\left(\mu_{1}\right)$ ist.

Beweis. Es treten hier Matrixelemente der Form

$$
(\Omega,[\widetilde{A}(\tilde{f}), C] \Omega)
$$

auf, die sich leicht auf die in Lemma 1 verwendete Form bringen lassen:

$$
\begin{gathered}
(\Omega, \tilde{A}(\tilde{f}) C \Omega)-(\Omega, C \tilde{A}(\tilde{f}) \Omega) \\
=\int \tilde{f}(-p) \psi(p) d \mu-\int \overline{\psi(-p)} \tilde{f}(p) d \mu,
\end{gathered}
$$

wobei $\psi(p)$ die $\mathscr{H}_{1}$-Komponente von $C \Omega$ ist. Mit Lemma 1 folgt die Behauptung.

Durch die Bedingung, daß $\tilde{\mathscr{D}}(T)$ dicht in $\mathscr{L}_{2}\left(\mu_{1}\right)$ liegt, wird das Maß $\mu_{1}$ bzw. $\mu$ festgelegt. Es ist jetzt noch zu zeigen, daß der Kommutator

$$
[A(g), C]
$$

selbst verschwindet, wenn das Matrixelement

verschwindet.

$$
(\Omega,[A(g), C] \Omega)
$$

Sei $A\left(x_{0}, \boldsymbol{f}\right)$ das verallgemeinerte freie Feld zum Maß $\mu$, das den Bedingungen aus Lemma 2 genügen möge. Das Feld sei in räumlicher Richtung schon mit einer Testfunktion $\boldsymbol{f}(\boldsymbol{x})$ mit kompaktem Träger verschmiert. Sei

$$
\begin{aligned}
\left(\Omega,\left[A\left(x_{0}, \boldsymbol{f}\right), C\right] \Omega\right) & =\left(\Omega, A\left(x_{0}, \boldsymbol{f}\right) C \Omega\right)-\left(\Omega, C A\left(x_{0}, \boldsymbol{f}\right) \Omega\right) \\
& =F^{+}\left(x_{0}\right)-F^{-}\left(x_{0}\right) .
\end{aligned}
$$

Lemma 3. Es gilt $F^{+}\left(x_{0}\right)-F^{-}\left(x_{0}\right)=0$ für alle $x_{0}$ genau dann, wenn $F^{+}\left(x_{0}\right) \equiv F^{-}\left(x_{0}\right) \equiv 0$.

Beweis. a) Gelte zunächst $F^{+}\left(x_{0}\right)-F^{-}\left(x_{0}\right)=0$ für alle $x_{0}$.

$$
F^{+}\left(x_{0}\right)=\left(\Omega, A\left(x_{0}, \boldsymbol{f}\right) C \Omega\right)=\left(\Omega, A(0, \boldsymbol{f}) U\left(-x_{0}\right) C \Omega\right)
$$

ist Randwert einer in der unteren Halbebene analytischen Funktion. Ebenso ist $F^{-}\left(x_{0}\right)$ Randwert einer in der oberen Halbebene analytischen Funktion. Da diese beiden Funktionen auf der reellen Achse stetig und identisch gleich sind, kann man sie zu einer ganz-analytischen Funktion erweitern. Nach einem bekannten Satz ist die Fouriertransformation einer ganzen Funktion ein Maß. Die Fouriertransformationen der komplexen Erweiterungen von $F^{+}$bzw. $F^{-}$haben ihren Träger in $\overline{V^{+}}$bzw. $\overline{V^{-}}$, 16 Commun.math. Phys.,Vol.11 
der gemeinsame Träger ist also nur der Nullpunkt im Impulsraum. Die Fouriertransformation der ganzen Funktion ist also ein $\delta$-Maß. Also ist

$$
F^{+}=F^{-}=\text {const. }
$$

Nach Konstruktion gilt für den Operator des verallgemeinerten freien Feldes

$$
\begin{aligned}
& \left(\Omega, A\left(f_{1}\right) \ldots A\left(f_{n}\right) C \Omega\right) \\
= & \left(\Omega, A\left(f_{1}\right) \ldots A\left(f_{n}\right)\left(E_{0}+E_{1}+\cdots+E_{n}\right) C \Omega\right),
\end{aligned}
$$

wobei $E_{i}$ der Projektor auf den Teilraum $\mathscr{H}_{i}$ von

ist. Damit ist

$$
\mathscr{H}=\bigoplus_{i=0}^{\infty} \mathscr{H}_{i}
$$

$$
F^{+}\left(x_{0}\right)=\left(\Omega, A\left(x_{0}, \boldsymbol{f}\right) C \Omega\right)=\left(\Omega, A\left(x_{0}, \boldsymbol{f}\right)\left(E_{0}+E_{1}\right) C \Omega\right)=\text { const. }
$$

Also müssen alle Beiträge bis auf den von $E_{0}$ verschwinden, d. h.

Analog für $F^{-}\left(x_{0}\right)$.

$$
F^{+}\left(x_{0}\right)=\left(\Omega, A\left(x_{0}, \boldsymbol{f}\right) \Omega\right)=0 .
$$

Wir redefinieren jetzt $C$ als

$$
C \rightarrow \bar{C}=C-(\Omega, C \Omega) \mathbf{1} .
$$

Kommutiert $C$ mit irgend einem Operator, so tut es natürlich auch $\bar{C}$. Wir setzen Lemma 2 voraus und beweisen

Lemma 4. $(\Omega,[A(f), C] \Omega)=0$ für supp $f$ beliebig dann und nur dann, wenn $\left(\Omega, A\left(f_{1}\right) \ldots A\left(f_{n}\right) \bar{C} \Omega\right)=0$ für alle $n$ und $\operatorname{supp} f_{i}$ beliebig.

Beweis. a) Sei zunächst $(\Omega,[A(f), C] \Omega)=0$.

Induktion: Für $n=1$ ergibt sich die Behauptung direkt aus Lemma 3 durch Integration mit einer zeitabhängigen Testfunktion.

$n=2$ : Wir betrachten Matrixelemente

$$
\left(\Omega, A(\varphi) A\left(f_{2}\right) C \Omega\right)
$$

mit $\varphi \in \mathscr{D}(T)$ und supp $f_{2}$ beliebig kompakt. Sei

Dann ist

$$
C_{2}:=A\left(f_{2}\right) \bar{C} .
$$

$$
\begin{aligned}
\left(\Omega,\left[A(\varphi), C_{2}\right] \Omega\right)= & \left(\Omega, A(\varphi) A\left(f_{2}\right) \bar{C} \Omega\right)-\left(\Omega, A\left(f_{2}\right) A(\varphi) \bar{C} \Omega\right) \\
& +\left(\Omega, A\left(f_{2}\right) A(\varphi) \bar{C} \Omega\right)-\left(\Omega, A\left(f_{2}\right) \bar{C} A(\varphi) \Omega\right) .
\end{aligned}
$$

Die beiden letzten Terme verschwinden nach Lemma 2 . Es bleibt

$$
\begin{aligned}
& \left(\Omega,\left[A(\varphi), A\left(f_{2}\right)\right] C \Omega\right)=[c-\mathrm{Zahl}](\Omega, \bar{C} \Omega) \\
= & {[c-\mathrm{Zahl}](\Omega, C \Omega)-[c-\mathrm{Zahl}](\Omega, C \Omega)=0 . }
\end{aligned}
$$

Wegen Lemma 2 verschwindet dieses Matrixelement auch für alle Testfunktionen $f_{1}$ mit beliebigem kompaktem Träger. Mit dem gleichen 
Argument wie in Lemma 3 folgt dann, daß sogar gilt

$$
\left(\Omega, A\left(f_{1}\right) C_{1} \Omega\right)=\left(\Omega, A\left(f_{1}\right) A\left(f_{2}\right) \bar{C} \Omega\right) \equiv 0 .
$$

Der Induktionsschluß von $n-1$ auf $n+1$ verläuft ganz analog, dabei wird wesentlich ausgenutzt, daß sich der Kommutator des verallgemeinerten freien Feldes als $c$-Zahl herausziehen läßt.

b) Die Gegenrichtung von Lemma 4 ist trivial.

Es gilt also $\bar{C}=0, \mathrm{~d}$. $\mathrm{h}$.

$$
C=(\Omega, C \Omega) \mathbf{1}=\lambda \cdot \mathbf{1} .
$$

$C$ kommutiert also mit allen Operatoren. Da das für beliebige Polynome von Feldern $A(f)$ bewiesen wurde, gilt

$$
[A(f), C] \equiv 0
$$

für alle Testfunktionen $f(x)$, denn die Testfunktionen mit kompaktem Träger bilden eine dichte Untermenge. Zusammenfassung von Lemma 2, 3 und 4 liefert den Beweis von Theorem 1.

Beweis von Theorem 2. Dazu benutzen wir in Lemma 2 Testfunktionen, deren Träger in zeitlicher Richtung $\delta$-förmig und in räumlicher Richtung kompakt ist. Die Menge ihrer Fouriertransformationen sei mit $\tilde{\mathscr{D}}(\delta)$ bezeichnet. Wir setzen voraus, daß

$$
\left[A\left(\delta\left(x_{0}=0\right), \boldsymbol{f}\right), C\right]
$$

verschwindet, zusammen mit allen zeitlichen Ableitungen. Es ergeben sich dann Terme der Form

$$
\int \psi(p) \tilde{f}(p) p_{0}^{k} d \mu_{1}, \quad \psi \in \mathscr{H}_{\mathbf{1}} .
$$

Es sei nun $\mu_{1}$ so ein Maß, daß das ZSP erfüllt ist für eine Zeitschicht $T$. Mit $f(x) \in \mathscr{D}(T)$ ist auch $e^{i z_{0} x_{0}} f(x) \in \mathscr{D}(T)$. Also läßt sich die Fouriertransformation

$$
\tilde{f}\left(p_{0}-z_{0}, \boldsymbol{p}\right) \in \tilde{\mathscr{D}}(T)
$$

zu einer ganzen Funktion erweitern und in eine Potenzreihe in $z_{0}$ entwickeln. Das Skalarprodukt mit einer beliebigen Funktion $\tilde{g}(p) \in L_{2}\left(\mu_{1}\right)$ ist

$$
\int \tilde{g}(p) \tilde{f}\left(p_{0}-z_{0}, \boldsymbol{p}\right) d \mu_{1}=\int \tilde{g}(p) \sum_{n}\left(p_{0}-z_{0}\right)^{n} \tilde{f}_{n}(\boldsymbol{p}) d \mu_{1} .
$$

Wegen des starken Abfalles der $\tilde{f}_{n}(\boldsymbol{p})$ und von $\mu_{\mathbf{1}}$ (s. [2]) konvergiert das Integral gleichmäßig zumindest in einer Umgebung der Ebene $p_{0}=z_{0}$. Also ergibt sich

$$
=\sum_{n} \int \tilde{g}(p) \tilde{f}_{n}(\boldsymbol{p})\left(p_{0}-z_{0}\right)^{n} d \mu_{1}(p) .
$$

Offenbar ist $\tilde{f}_{n}(\boldsymbol{p})\left(p_{0}-z_{0}\right)^{n} \in \tilde{\mathscr{D}}(\delta)$. Ist $\tilde{g}(p)$ eine beliebige zu $\tilde{\mathscr{D}}(\delta)$ orthogonale Funktion, so verschwindet jeder Summand in (2) und damit $16^{*}$ 
auch das Integral (1). $\tilde{g}(p)$ ist also auch zu $\tilde{\mathscr{D}}(T)$ orthogonal. Da $\tilde{\mathscr{D}}(T)$ dicht in $\mathscr{L}_{2}\left(\mu_{1}\right)$ ist, trifft das auch für $\tilde{\mathscr{D}}(\delta)$ zu. Alle Feldoperatoren lassen sich also durch Testfunktionen zu einer scharfen Zeit erzeugen.

Herrn Professor Dr. H. J. Borchers danke ich herzlich für wertvolle Hinweise und Diskussionen.

\section{Literatur}

1. Borchers, H. J.: Three remarks on quantum field theory. New York University Progress Report 1963.

2. HaAG, R., and B. Schroer: J. Math. Phys. 3, 248 (1962).

3. Greenbera, O. W.: Ann. Phys. 16, 158 (1961).

4. Jost, R.: The general theory of quantized fields. Am Math. Soc. Providence: 1965.

C. Möllenhoff

Universitäts-Sternwarte

3400 Göttingen, Geismarlandstr. 11 